\title{
Experiencia metacompleja para la construcción de un modelo de diagnóstico médico por estudiantes
}

\author{
Juan M. Muñoz-Cano
}

Introducción. Se desarrolló este trabajo en base a proyectos para elaborar una estrategia de diagnóstico, con eje en 'diarrea', causa importante de morbilidad y mortalidad en México, pues la educación médica orientada a las asignaturas desde el paradigma de la 'transmisión' no forma la competencia.

Sujetos y métodos. Se trabajó con 16 estudiantes de medicina de una universidad del sureste de México y se estructuró en base al modelo de experiencia metacompleja 'El diálogo nos hace escribir nueva teoría'. El producto fue la elaboración de un proceso diagnóstico para identificar el mecanismo del síntoma y las causas de mayor probabilidad. Los avances se retroalimentaron de acuerdo con una matriz de evaluación y la acreditación se obtuvo al cumplir el 80\% de los criterios de la matriz.

Resultados. La mitad de los estudiantes terminaron la experiencia. Aprendieron a identificar patrones de conducta sin utilidad para el diagnóstico y a evaluar la relevancia de los contenidos de los textos escolares y la necesidad de buscar información en bibliotecas virtuales. Tras construir sus conceptos, que se evidenciaron mediante diagramas de flujo y cuadros comparativos, pudieron manejar guías clínicas y revisiones sistemáticas, así como las normas oficiales mexicanas.

Conclusión. Mediante el proceso de la construcción del cuerpo metacomplejo, los estudiantes ponderaron de manera positiva su papel activo y pudieron desarrollar un pensamiento crítico.

Palabras clave. Aprendizaje basado en proyectos. Aprendizaje situado. Educación médica. Metacognición.

\section{Metacomplex experience for the construction of a model of medical diagnosis by students}

Introduction. The aim of this study based on projects was develop a strategy for diagnosis. Axis was 'diarrhea', a major cause of morbidity and mortality in Mexico, because medical education from 'transmission' paradigm can't construct the competence.

Subjects and methods. We worked with 16 medicine students at a university in southern Mexico and development was based on the model of metacomplex experience 'Dialogue makes us write new theory'. The obtained product was a diagnostic process to identify the symptoms mechanism and causes more likely of diarrhea. Progress was evaluated according to a rubric and accreditation to get $80 \%$ of criteria met.

Results. Half of the students completed the experience. They learned to identify patterns of behavior without useful for diagnosis, to assess the relevance of the contents of textbooks and the need to seek information in virtual libraries. After construct their concepts, made transparent through flow charts and comparative tables, they could handle clinical guidelines and systematic reviews as well as the Mexican official standards.

Conclusion. Through the process of building the metacomplex body, students positively weighted active role and could develop critical thinking.

Key words. Medical education. Metacognition. Project-based learning. Situated learning.

\section{Introducción}

En el esquema tradicional de la educación superior se trabaja en base a la exposición, memorización y aplicación de exámenes tipo test [1,2]. En ese modelo hay disociación entre las clases teóricas y las clases prácticas, lo que no permite una respuesta estructurada a la resolución de problemas individuales ni sociales. En la educación médica, esta pedagogía no promueve que el estudiante construya significativamente conceptos y los aplique en su contexto. Por otra parte, los profesores de medicina
División Académica de Ciencias de la Salud. Universidad Juárez Autónoma de Tabasco. Villahermosa, Tabasco, México.

Correspondencia: Dr. Juan Manuel Muñoz Cano. División Académica de Ciencias de la Salud. Universidad Juárez Autónoma de Tabasco. Avda. Méndez, 2838. Tamulté, Villahermosa, Tabasco, México. CP 86150.

E-mail:

juan.munoz@ujat.mx

Agradecimientos:

A G.A. Luque, G.A. Alegría, U.O. Ríos, B. Pineda, G.A. Damián, J.C. Hernández, G. Díaz y J.F. Villar, por su participación como estudiantes en este proyecto; a T.N.J. Maldonado, por verificar los avances de acuerdo con la matriz de evaluación.

Conflicto de intereses: No declarado.

Conflict of interests: None declared.

(c) 2012 Educación Médica 
apoyan su enseñanza en una visión reducida de la enfermedad, por lo que priorizan actividades mecánicas y estereotipadas en los hospitales de segundo y tercer nivel. Para superar esto, se han analizado los perfiles, aprendizajes y roles de los licenciados en base a competencias, que se han diseñado en genéricas, transversales y profesionales [3], pues 'la capacidad analítica y sintética, su aplicación a la resolución de problemas y a la consecuente toma de decisiones' debería ser el eje de la actividad diaria del médico [4]; por consiguiente, es necesario transformar la práctica educativa $[1,2]$.

En la educación médica, el aprendizaje basado en proyectos es una técnica que permite integrar conceptos que se encuentran más allá de los textos escolares. Los proyectos se relacionan con la manera en que se genera el conocimiento en los sitios de producción [5]. Los centros de investigación no se limitan a los contenidos de una asignatura, sino que trabajan en la resolución de problemas por medio de proyectos. El trabajo basado en proyectos constituye una tendencia que se encuentra explícita en muchos programas y modelos educativos; sin embargo, se han incorporado escasamente a la práctica escolar en todos los niveles.

El aprendizaje basado en proyectos se sustenta en lo que la evidencia científica explica cómo se aprende [6]. En contraste con la idea de que el aprendizaje es lineal y que unos conceptos deben aprenderse antes que otros, se ha encontrado que es necesario plantearse un problema o cuestión para resolverlo y construir un marco procedimental y conceptual más complejo. En la medida en que el estudiante tiene más elementos cognitivos, puede comprender lo que se tiene que resolver en su contexto social y cultural. 'La idea fundamental es el diseño de un planteamiento de acción donde los estudiantes identifican factores de riesgo a enfrentar, medidas alternativas para asegurar el éxito, no la solución de problemas simples o realización de actividades' [7]. Aunque en varias instituciones de educación superior la educación médica transita hacia el aprendizaje basado en problemas, no hay experiencias documentadas del aprendizaje de la medicina basado en proyectos. Por eso, con el propósito de sistematizar una experiencia basada en el paradigma de la complejidad, se planteó el desarrollo de un curso donde se conjuntaran diversas asignaturas y disciplinas articuladas a partir de preguntas eje: ¿cómo instrumentar un proceso basado en problemas más allá de la simple resolución de un problema?, ¿cómo transitar desde un papel pasivo hacia uno donde los estudiantes modifiquen sus nociones y conceptos y desaprenden para aprender?, ¿qué ayudas necesitan los estudiantes para asumir un papel activo en la construcción de conceptos complejos?

\section{Sujetos y métodos}

Con el propósito de realizar una propuesta educativa centrada en proyectos se desarrolló una intervención educativa con estudiantes de medicina de una universidad del sureste de México. Se partió de que una intervención educativa es un proceso amplio y complejo, surgido desde los docentes y su reflexión de la práctica (acciones, relaciones y significaciones); busca detectar problemáticas, explicarlas y proponer alternativas innovadoras de transformación. La intervención contó con la autorización del comité local de investigación.

\section{Muestra}

Se trabajó con 16 estudiantes, con distintos grados de avance, que podían inscribirse de manera semiflexible en un curso de agosto a diciembre del 2010. En el grupo original había estudiantes que ingresaron en la escuela de medicina entre agosto del 2000 y agosto del 2009, ya que el ingreso es anual. Seis de ellos se dieron de baja en el transcurso del primer mes y otros dos abandonaron en el siguiente. Quienes permanecieron ingresaron en el año $2000(n=1)$, $2007(n=1), 2008(n=5)$ y $2009(n=1)$. De esta manera, los estudiantes tenían distintas trayectorias escolares y diferentes asignaturas cursadas.

\section{Diseño del curso}

Se diseñó el curso basado en proyectos y se consideraron dos aspectos cruciales de una intervención de este tipo: suponer la solución de un problema donde -no necesariamente- la estructura es definida por los estudiantes, y debe haber un producto, sea una tesis, informe, modelo o diseño [8]. En este caso, la construcción cognitiva surgió de la revisión de la bibliografía [9]. De acuerdo con la clasificación de Kolmos [10], se trató de un proceso por problemática. Los estudiantes no tenían aproximaciones a los métodos para sistematizar la realización de un diagnóstico ni los problemas primordiales hacia los cuales deberían dirigir sus esfuerzos. De esta manera, la problemática dirigió el proyecto. El proyecto se estructuró con base en el modelo de experiencia metacompleja 'El diálogo nos hace escribir nueva teoría', cuyas etapas son: motivación-introducción, cuerpo metacomplejo, avance temático y plantea- 
miento de problemas, y construcción cognitiva [9]. Las etapas planeadas para la experiencia fueron:

- Construcción de la idea central.

- Contraste del conocimiento previo con la idea central.

- Reflexión y etapa de construcción de la propia teoría.

- Apropiación teórica.

Los avances se evaluaron de acuerdo con una matriz de evaluación que se distribuyó entre los estudiantes en formato electrónico en la guía del curso.

\section{Procedimiento}

La motivación-introducción para la construcción de la idea central se basó en el perfil epidemiológico y en la segunda causa de atención médica, y de mortalidad en niños menores de 5 años en México, las enfermedades diarreicas. A los estudiantes se les pidió elaborar un esquema en el que se incorporaran las clasificaciones del problema, y un proceso para identificar el mecanismo y las causas de mayor probabilidad. Se discutieron con los estudiantes los conceptos que usaban de manera rutinaria y que necesitaban deconstruirse-reconstruirse, así como la necesidad de desaprender otros, y se les proporcionaron apoyos didácticos que se diseñaron como parte de la retroalimentación formativa (Figura). La acreditación se realizó de acuerdo con Díaz-Barriga [1] y se obtuvo una vez cumplidos el $80 \%$ de los criterios especificados en la matriz de evaluación para el producto completo, o al menos todos los indicadores en el nivel 2 (Tabla). Para ello, cada estudiante tuvo que incorporar las observaciones a sus informes y presentar otros sustancialmente diferentes en cada ocasión. Esto se realizó con el propósito de cambiar el enfoque de la práctica ordinaria basada en la respuesta correcta en cuestiones puntuales, característica de los exámenes tipo test [11], hacia una más centrada en el desempeño [12].

\section{Resultados}

\section{Motivación-introducción}

Se construyeron conceptos en torno al diagnóstico nosológico de la diarrea, ya que se revisaron los motivos de atención, la morbilidad y la mortalidad asociados a diarrea como dato clínico de mayor relevancia. Para la identificación de sus necesidades de desaprendizaje-aprendizaje se realizó la evaluación previa, donde las preguntas-eje que harían a
Figura. Retroalimentación para la construcción del árbol de problemas y el árbol de soluciones.

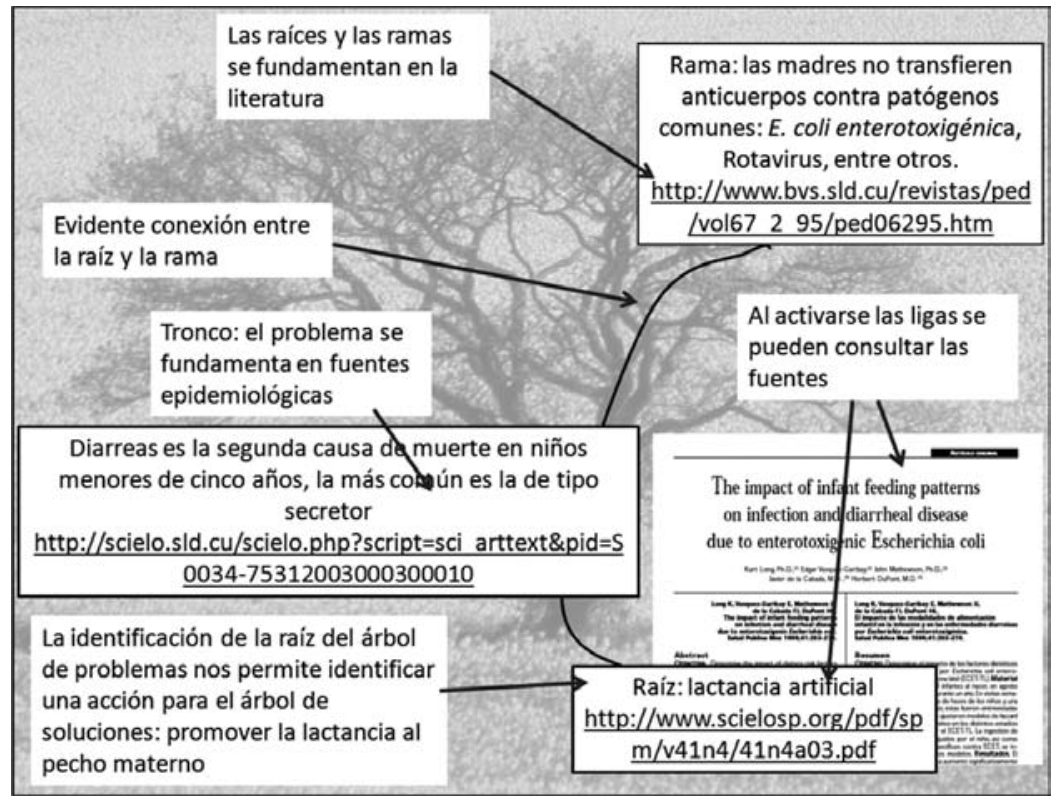

sus pacientes en caso de diarrea serían: ‘qué fue lo último que comió?' (A1), ‘qué alimentos consumió ayer?' (D1), '¿qué ha comido?' (G1). Se discutió con ellos que, aun cuando no se recuerda el origen, la cosmovisión de las etnias locales clasifica los alimentos en fríos y calientes (no su temperatura o modo de preparación), pues explica posibilidades de enfermar por esta causa. Sin embargo, ni los estudiantes ni los médicos en servicio que hacen esa pregunta -observación hecha posteriormente por los estudiantes- identifican el origen y la utilidad social de la misma, pues toma en cuenta la cultura popular. Un segundo aspecto para desaprenderaprender fue que los estudiantes, sin tener un método para realizar un diagnóstico (de sindromático a nosológico o etiológico), en la manera que han observado realizan sus diagnósticos. Así, los médicos en servicio confunden las causas con los efectos: 'puede ser un rotavirus' $(\mathrm{C} 1)$, 'una infección gastrointestinal' (D1, F1). Se discutieron los datos de las tablas epidemiológicas disponibles en la página web de la Secretaría de Salud de México, donde se observan diagnósticos como gastroenteritis, amibiasis intestinal, salmonelosis, pero no síndrome de intestino irritable, a pesar de que en la revisión de la bibliografía se encontró que un $9 \%$ de todas las consultas podrían ser por esta causa [13]. 
Tabla. Matriz de evaluación en la elaboración de la fase de problematización.

\begin{tabular}{|c|c|c|c|c|}
\hline $\begin{array}{l}\text { Identificación } \\
\text { del problema }\end{array}$ & $\begin{array}{l}\text { Describe una situación } \\
\text { que origina un problema } \\
\text { y sus consecuencias }\end{array}$ & $\begin{array}{l}\text { Relaciona un problema } \\
\text { con alguna de sus causas }\end{array}$ & $\begin{array}{l}\text { Menciona una situación } \\
\text { que es un problema de } \\
\text { salud }\end{array}$ & $\begin{array}{l}\text { Sólo menciona temas } \\
\text { sin problematizar }\end{array}$ \\
\hline $\begin{array}{l}\text { Redacción de } \\
\text { la definición } \\
\text { del problema }\end{array}$ & $\begin{array}{l}\text { Elabora un escrito fluido } \\
\text { y sustentado en datos } \\
\text { conceptuales y numéricos }\end{array}$ & $\begin{array}{l}\text { Elabora un escrito donde se } \\
\text { pueden identificar causas y } \\
\text { efectos del problema }\end{array}$ & $\begin{array}{l}\text { Elabora un escrito donde no } \\
\text { interrelaciona los conceptos } \\
\text { para apoyar la argumentación }\end{array}$ & $\begin{array}{l}\text { Se limita a transcribir } \\
\text { fragmentos de los } \\
\text { textos que consulta }\end{array}$ \\
\hline $\begin{array}{l}\text { Identificación } \\
\text { del dilema }\end{array}$ & $\begin{array}{l}\text { Identifica varias posiciones, } \\
\text { toma una con respecto al } \\
\text { problema y sustenta por } \\
\text { qué no elige otras }\end{array}$ & $\begin{array}{l}\text { Toma una posición respecto } \\
\text { del problema, pero no } \\
\text { identifica otras facetas } \\
\text { de éste }\end{array}$ & $\begin{array}{l}\text { Elabora definiciones } \\
\text { relacionadas con el problema, } \\
\text { pero no identifica diversas } \\
\text { posiciones }\end{array}$ & $\begin{array}{l}\text { Aborda el problema con } \\
\text { una visión limitada que } \\
\text { no identifica posiciones } \\
\text { respecto a éste }\end{array}$ \\
\hline $\begin{array}{l}\text { Elaboración de } \\
\text { conclusiones y } \\
\text { recomendaciones }\end{array}$ & $\begin{array}{l}\text { Sustenta las conclusiones } \\
\text { en el análisis de la } \\
\text { información consultada }\end{array}$ & $\begin{array}{l}\text { Escribe enunciados que no } \\
\text { expresan conclusiones y } \\
\text { recomendaciones puntuales }\end{array}$ & $\begin{array}{l}\text { Enuncia ideas inconexas } \\
\text { relacionadas con el problema }\end{array}$ & $\begin{array}{l}\text { Sus enunciados no } \\
\text { contienen información } \\
\text { relevante respecto al } \\
\text { problema }\end{array}$ \\
\hline $\begin{array}{l}\text { Ortografía } \\
\text { y gramática }\end{array}$ & $\begin{array}{l}\text { Comete un máximo de } \\
\text { tres errores ortográficos }\end{array}$ & $\begin{array}{l}\text { Comete un máximo de } \\
\text { diez errores ortográficos }\end{array}$ & $\begin{array}{l}\text { Comete un error } \\
\text { ortográfico por línea }\end{array}$ & $\begin{array}{l}\text { No sigue las reglas } \\
\text { ortográficas }\end{array}$ \\
\hline
\end{tabular}

Después se acordó la elaboración de un producto final consistente en un diagrama de flujo o una secuencia de pasos para identificar de manera más precisa un diagnóstico nosológico, un uso más racional de los antibióticos y la necesidad de evaluar la sensibilidad y especificidad del estudiante en la valoración de los datos clínicos.

\section{Cuerpo metacomplejo}

Durante el curso se analizaron las diferencias entre esta propuesta y las actividades que habían realizado hasta el momento, donde la práctica dominante es la exposición de temas, por parte del alumno, de manera secuencial. Se discutió con los estudiantes acerca de la plasticidad cerebral, la regeneración neuronal y las neuronas en espejo, con el auxilio de recursos en línea como vídeos de Eduard Punset, y lecturas como el capítulo 1 del libro Cómo aprende la gente [6] (se encuentra en castellano en www. eduteka.org). Se hizo énfasis en que los adultos aprendemos en base a un problema que se debe resolver, por lo que es crucial el manejo de bases de datos, bibliotecas virtuales y, sobre todo, la búsqueda de información en fuentes de asignaturas diferentes. Se insistió en la necesidad de plantear hipó- tesis diagnósticas, probabilidades, diagnósticos alternativos y posibilidades, como elementos de un proceso de atención que se desarrolla en la incertidumbre, pero que puede hacerse evidente. Al finalizar el curso, un estudiante dijo: 'lo que más me impactó fue cuando dijo que ustedes [los profesores] sólo son mediadores del conocimiento y de nosotros depende lo demás' (G6).

\section{Avance temático orientado hacia la construcción}

Al revisar la bibliografía, los estudiantes registraron observaciones relevantes:

- La mayoría de los autores de los textos escolares raramente dan importancia a las diarreas y no es inusual encontrar menos de una página para las de origen vírico [14], que en México son causa principal de deshidratación y muerte.

- Se describen pobremente las diferencias entre los distintos tipos de acuerdo con los mecanismos que las producen (secretora, exudativa, osmótica, malabsorción, motilidad), o como parte de los problemas que se asocian a fiebre $[15,16]$; otros se limitan a problemas agudos [17].

- Pueden tener apartados acerca de los problemas crónicos, aunque centrados en las causas y no en 
los procesos para evaluar las diferencias; por ejemplo, se cita que el síndrome de intestino irritable es un problema funcional [16], pero no se explica el significado fisiopatológico ni clínico de 'funcional.'

- En la bibliografía, incluso la mexicana, se denomina 'diarrea del viajero' [18] a lo que para los estudiantes debe ser un problema habitual, de acuerdo con los datos epidemiológicos: la gastroenteritis aguda. También se persiste en asociarla a fiebre tifoidea [19], lo que no tiene sustento clínico.

\section{Construcción cognitiva}

Al finalizar el curso se obtuvieron resultados desde esquemas comparativos con el propósito de elaborar un cuadro basado en las clasificaciones (tiempo, localización, mecanismo) para construir una hipótesis diagnóstica con mayor especificidad, así como diagramas de flujo para la construcción de diagnósticos nosológicos a partir de síntomas y datos que antes percibían como inconexos, de acuerdo con un contexto de desempeño que incluye edad, sexo y factores de riesgo. Sus informes iniciales eran meras copias de información disponible en los motores habituales (principalmente Google), desde donde pasaron a la construcción de comentarios sustentados en la información de bibliotecas virtuales, aun cuando ello requiere un mejor dominio de las fuentes: 'seguimos fomentando el uso de antibióticos con casi todos los pacientes que presentan una enfermedad diarreica, sin hacer la anamnesis correcta ni un diagnóstico certero y, por lógica, erramos en el tratamiento, que dependerá del mecanismo que cause la diarrea. El principal tratamiento, que es evitar la deshidratación, se deja como un tratamiento secundario' (G5).

\section{Discusión}

Sea porque los estudiantes estaban acostumbrados a un papel pasivo en las actividades escolares y no deseaban salir de esa zona de confort [4], o por la angustia que representaba intentar otra estrategia tras muchos años en el sistema escolar [9], de 16 alumnos inscritos en el curso, seis se dieron de baja en el tiempo que la Administración tiene asignado para ello; otros dos estudiantes abandonaron posteriormente y prefirieron que se les otorgara una calificación reprobatoria. Ello evidencia la necesidad de trabajar con los estudiantes para la construcción de estrategias de afrontamiento para el desarrollo de proyectos y la resolución de problemas, lo cual es una de las competencias transversales de la educación superior [2].

En el proceso de desaprender-aprender necesario para construir una interpretación de los hechos, es preciso identificar los conocimientos previos del estudiante y que el docente facilite las nuevas construcciones conceptuales [6]. Esto se hizo evidente en la primera intervención de los estudiantes, con preguntas como: '¿qué fue lo que comió?'. Éstas se formularon a pesar de la escuela, o precisamente por el tipo de proceso escolar orientado a la pasividad del alumno [20]. En sus otras actividades escolares en primero y segundo nivel de atención, los estudiantes observaron, y comentaron en esa clase, una práctica médica de tipo anecdótico que contrasta con la basada en una experiencia sustentada en el conocimiento científico. En esa práctica no se tienen en consideración normas ni guías clínicas -a pesar de que son un proyecto nacional [21]- para la prescripción de medicamentos o para el proceso de educar a los pacientes y sus familias, lo que se traduce en la persistencia del mal uso de antibióticos y antitusígenos [22], entre otros, lo cual fue identificado por los estudiantes que terminaron este curso.

De acuerdo con la manera habitual de analizar los avances de los estudiantes (cursar la asignatura A, luego la B, etc.), sería de esperar que estudiantes con más asignaturas cursadas respondieran mejor en el proyecto; sin embargo, se observó que los estudiantes con menos semestres cursados respondieron mejor para 'usar, en nuevos escenarios, lo que hayan aprendido' [6]. Esto sustenta la observación de que el modo cómo funciona la escuela deforma y no contribuye a la construcción de competencias y capacidades para el aprendizaje autónomo.

Es importante considerar que, según Edgar Morin [23], 'el pensamiento complejo está animado por una tensión permanente entre la aspiración a un saber no parcelado, no dividido, no reduccionista, y el reconocimiento de lo inacabado e incompleto de todo conocimiento'. En este sentido, el propósito de la innovación no puede circunscribirse a una asignatura en particular, $o$ a las dificultades que dentro de una asignatura plantea la memorización de determinados conceptos o paradigmas. Plantea la necesidad de una innovación que, dentro de los límites impuestos por las asignaturas, se pueda transitar a una práctica docente que, sin modificaciones curriculares de fondo -ya que la flexibilidad administrativa aún no es una realidad en nuestras instituciones-, debilite los límites al desarrollar un proceso situado, centrado en situaciones de la vida real, y no en ejercicios de empleo de las tecnologías de la 
información y la comunicación, puesto que ni las asignaturas ni los textos de las asignaturas reproducen el ámbito de producción del conocimiento.

En conclusión, uno de los logros de la experiencia educativa metacompleja es que los estudiantes aprenden a evaluar sus avances y sus construcciones conceptuales en nuevas situaciones.

Es necesario desarrollar estrategias motivacionales para que los estudiantes elaboren objetivos y desarrollen estrategias con que cumplir sus metas académicas en nuevos escenarios presenciales y virtuales.

Las actividades pasivas no confieren las capacidades necesarias para que los estudiantes sean aprendices autónomos y puedan resolver problemas de manera independiente; por lo contrario, los hacen dependientes del profesor.

En las instituciones de educación superior que mantienen el esquema curricular centrado en asignaturas, es necesario desarrollar un proceso escolar que las integre en base a situaciones de la vida real, para superar la actividad centrada en el libro escolar de asignatura.

\section{Bibliografía}

1. Díaz-Barriga AR. El docente y los programas escolares. Lo institucional y lo didáctico. México: IISUE; 2009.

2. Agencia Nacional de Evaluación de la Calidad y Acreditación. El profesional flexible en la sociedad del conocimiento. Informe ejecutivo. 2008. URL: http://www.aneca.es/media/ 158162/informeejecutivoaneca_jornadasreflexv20.pdf.

3. Abreu LF, Cid-García A, Herrera-Correa G, Lara-Vélez V Laviada R, Sánchez-Aparicio JA. Perfil por competencias del médico general mexicano. México: AMFEM; 2008. URL: http://www.amfem.edu.mx/intranet/descargas/competencias.pdf.

4. Baños J, Pérez J. Cómo fomentar las competencias transversales en los estudios de ciencias de la salud: una propuesta de actividades. Educ Med 2005; 8: 216-25.

5. Thomas JW. A review of research on project-based learning. URL: http://www.bobpearlman.org/BestPractices/PBL Research.pdf.

6. Committee on Developments in the Science of Learning with additional material from the Committee on Learning Research and Educational Practice, National Research Council. How people learn: brain, mind, experience, and school. Expanded edition. Washington: National Academy Press; 2000. URL: http://www.nap.edu/catalog.php?record_id=9853.

7. Galeana L. Aprendizaje basado en proyectos. CEUPROMED 2009. URL: http://ceupromed.ucol.mx/revista/PdfArt/1/27.pdf.

8. Helle L, Tynjälä P, Olkinoura E. Project-based learning in post-secondary education. Theory, practice and rubber sling shots. High Educ 2006; 51: 287-314.

9. González-Velasco JM. El bucle educativo, pensamiento complejo y transdiciplinariedad. [monografía en Internet]. Comunidad Pensamiento Complejo. URL: http://www. pensamientocomplejo.com.ar/docs/files/Dr.\%20Juan\%20 Miguel\%20Gonzlez\%20Velasco\%20PhD,\%20El\%20bucle\%20 educativo-\%20Aprendizaje,\%20Pensamiento\%20Complejo $\% 20 y \% 20$ Transdisciplinariedad.pdf.

10. Kolmos A. Estrategias para desarrollar currículos basados en la formulación de problemas y organizados en base a proyectos. Educar 2004; 33: 77-96.

11. Pozuelos-Estrada FJ, Alonso-Martín P, Boza-Carreño A, Conde-Rodríguez A, Monescillo-Palomo M, Romero-Muñoz A. La evaluación formativa: una propuesta para transformar la enseñanza en el ámbito universitario. In ContrerasGonzález LC, Rodríguez-López JM, Morales-Gil FJ, eds. Innovamos juntos la universidad. Huelva: Universidad de Huelva; 2006. p. 235-51.

12. Harden RM, Crosby JR, Davis MH, Friedman M. Outcome based education from competency to meta-competency: a model for the specification of learning outcomes. Med Teach 1999; 21: 546-52.

13. Tort S, Balboa A, Marzoc M, Carrillo R, Mínguez M Valdepérez J, et al. Guía de práctica clínica sobre el síndrome del intestino irritable. Gastroenterol Hepatol 2006; 29: 467-521.

14. Koo H, Shandera WX. Infecciones por virus y ricketsias. In McPhee SJ, Papadakis MA, eds. Diagnóstico clínico y tratamiento de Lange. México: McGraw-Hill; 2008. p. 1178-226

15. Weber R, Fontana A. Fiebre. In Siegenthaler W, ed. Diagnóstico diferencial en medicina interna. México: Manual Moderno; 2007. p. 106-56.

16. Fried M, Bauerfiend P, Fox M, Muellhaupt B. Diarrea. In Siegenthaler W, ed. Diagnóstico diferencial en medicina interna. México: Manual Moderno; 2007. p. 808-27.

17. Mota S. Diarrea. In Narro-Robles J, Rivero-Serrano O, LópezBárcena JJ, eds. Diagnóstico y tratamiento en la práctica médica. México: Manual Moderno; 2008. p. 97-108.

18. Jacobs RA, Guglielmo BJ, Chin-Hong PV. Problemas comunes de las enfermedades infecciosas y tratamiento antimicrobiano. In McPhee SJ, Papadakis MA, eds. Diagnóstico clínico y tratamiento de Lange. México: McGraw-Hill; 2008. p. 1103-49.

19. Higuera-Ramírez FJ. Fiebre tifoidea y otras salmonelosis. In Narro-Robles J, Rivero-Serrano O, López-Bárcena JJ, eds. Diagnóstico y tratamiento en la práctica médica. México: Manual Moderno; 2008. p. 797-801.

20. Loría-Castellanos J, Márquez-Ávila G, Valladares-Aranda MA. Aptitud clínica ante el paciente pediátrico con asma grave en residentes de pediatría y urgencias. Educ Med 2010; 13: 247-54.

21. Torres-Arreola LP, Peralta-Pedrero ML, Viniegra-Osorio A, Valenzuela-Flores AA, Echeverría-Zuno S, Sandoval-Castellanos FJ. Proyecto para el desarrollo de guías de práctica clínica en el Instituto Mexicano del Seguro Social. Rev Med Inst Mex Seguro Soc 2010; 48: 661-72.

22. Rendón-Macías ME, Hernández-Romano FA, IglesiasLeboreiro J, Bernárdez-Zapata I. Conducta terapéutica en niños atendidos en urgencias durante la epidemia de influenza AH1N1. Rev Med Inst Mex Seguro Soc 2010; 48: 607-14.

23. Morin E. Introducción al pensamiento complejo. Física RU. URL: http://www.fisica.ru/dfmg/teacher/archivos/ Introduccion_al_pensamiento_complejo_Edgar_Morin.pdf. 